\title{
High Mobility Group A2 (HMGA2) and Matrix Metalloproteinase 12 (MMP12) Genes Expression in Egyptian Patients with Laryngeal Squamous Cell Carcinoma
}

\author{
Original \\ Article \\ Diaa El-Din Mohamed El-Hennawi', Mohamed Tawfik El Tabbakh ${ }^{1}$, Nagwan Abd El-Aziz \\ Sabek $^{2}$, Ashraf Saad Abou-Halawa ${ }^{1}$, Amr Mohammed Fareed ${ }^{1}$ \\ ${ }^{1}$ Otorhinolaryngology department, ${ }^{2}$ Medical Biochemistry and Molecular Biology \\ department, Faculty of Medicine, Suez Canal University, Ismailia, Egypt.
}

\begin{abstract}
Background: genetic studies of laryngeal squamous cell carcinoma (LSCC) are rare in Egypt. Increased expression of some relevant genes may have diagnostic and therapeutic implications.

Aim of the study: to compare the expression of HMGA2 and MMP12 genes in LSCC tumor tissue with their expression in adjacent non-tumor tissue.

Type of the study: Descriptive study

Setting: Tertiary referral hospital-Ismailia-Egypt

Patients and Methods: 24 male patients (age range 49-78 years) with LSCC were included in the study. Under general anesthesia, two biopsies were taken: one from the tumor tissue and the other from the adjacent normal tissue and they were examined histopathologically and then analyzed for level of expression of MMP12 and HMGA2 genes.

Results: Median expression levels of HMGA2 and MMP12 in the whole group were 0.98 and .8 (fold change) in normal tissue and 6.49 and 122.6 (fold change) in tumor tissue respectively. There was higher expression of the HMGA2 and MMP12 genes in tumor tissue compared to normal tissue $(P<0.0001)$. Also there was positive correlation of level of expression of the two genes with the degree of tumor differentiation $(P<.0001$ and $\mathrm{p}<.0008)$

Conclusion: Expression of HMGA2 and MMP12 genes is higher in LSCC compared to normal tissue samples. The higher the level of expression of the two genes, the poorer the degree of differentiation of the tumor Further studies should be pursued to determine the diagnostic, therapeutic and prognostic implications of this in management of LSCC.
\end{abstract}

Key Words: Carcinoma, HMGA2, laryngeal, MMP12.

Received: 27 November 2018, Accepted: 18 May 2019

Corresponding Author: Ashraf Saad Abou-Halawa, MD, Otorhinolaryngology department, Faculty of Medicine, Suez Canal University, Ismailia Egypt, Tel.: 01013834342, E-mail: ashraf_halawa@yahoo.com

ISSN: 2090-0740, July 2019 Vol.20, No.2

\section{INTRODUCTION}

Laryngeal squamous cell carcinoma (LSCC) has a yearly incidence of about 540,000 patients worldwide with almost $50 \%$ mortality rate ${ }^{[1]}$. Its incidence is more in men than in women (about 5:1). Smoking and alcoholism are the most known risk factors ${ }^{[2]}$.

Although early diagnosis and management of LSCC improve disease prognosis, the survival rate is almost the same during the last two decades ${ }^{[3]}$. Studying the genetic alteration in the tumor tissue may lead to a breakthrough in its management ${ }^{[4]}$. Applying molecular biology methods may help in the diagnostics, choice of optimal course of treatment and in determining the extent of surgical procedure.

Many genes showed change in their expression level in squamous cell carcinoma of head and neck $(\mathrm{SCCHN})^{[5]}$. High Mobility Group AT-hook 2 (HMGA2) gene [location: 12q15] encodes a transcriptional regulating factor ${ }^{[6]}$ but also it controls the expression of some tumor suppressors by a negative feedback. It has been proposed as an indicator for tumor prognosis and its over-expression has been linked to diminished patients' survival ${ }^{[7]}$.

Matrix Metalloproteinase-12 (MMP12) [location: 16q12.2] is a zinc dependent enzyme that can split the constituents of the extracellular matrix and it may be activated either intracellularly or extracellularly. It plays a role in adjustment of vascularity and breakdown of extracellular matrix e.g. in the endometrium during menstruation $^{[8]}$. Because it can disturb the basement membrane, it may play a role in the malignant growth, tissue invasion and metastasis ${ }^{[9]}$.

In Egypt, still no genetic studies of LSCC have been done. In the current research, we studied the pattern of expression of HMGA2 and MMP12 genes in grossly tumorous tissue and in apparently normal surrounding laryngeal mucosa. 
This helps to understand the clinical behavior of this tumor and may lead to improvement in diagnosis and treatment of this disease.

\section{SUBJECTS AND METHODS}

\section{Ethical consideration:}

This study was approved by institutional ethics review board in the Faculty of Medicine-Suez Canal University. Written consent was obtained from each patient before inclusion in the study.

Subjects: Twenty four patients with LSCC presenting to Otorhinolaryngology department-Suez Canal University Hospital- Ismailia-Egypt in the period of April 2016 to December 2017 were included in the study. Excluded from the study were patients with previous head and neck irradiation or chemotherapy and those with recurrent disease.

\section{Methods}

All patients were initially evaluated through history taking and clinical examination including telescopic and flexible laryngoscopy in the clinic. Then $\mathrm{CT}$ was requested. This was done before the patient was scheduled for direct laryngoscopy under general anaesthesia. During this procedure, microlaryngoscopy and angled telescopes were used for thorough assessment of the lesion. To confirm the diagnosis and for the purpose of this research, two biopsies were taken: Biopsy I from the tumor itself and Biopsy 2 from a nearby endolaryngeal or hypopharyngeal mucosa 1 $\mathrm{cm}$ away from the tumor.

Using separate sterile microforceps, two bunches were taken for each biopsy: one bunch submerged in 10\% formalin and the other was immediately submerged in RNA stabilizing Reagent (Cat \# 76104; Qiagen, Hilden, Germany) in Cryotubes and was kept in $-80^{\circ} \mathrm{C}$ freezer for RNA isolation. Histopathological examination was done for biopsy I and II. Tumor was classified into three grades: highly differentiated, moderately differentiated and poorly differentiated In case of histopathology being negative for $\mathrm{SSC}$, the frozen tissues were discarded sanitarily.

\section{Molecular laboratory investigations:}

\section{Extraction of RNA}

RNA was extracted from the frozen cancerous and normal tissues according to the method used by Mohan et al., (2006), using QIAMP RNA Blood Mini Kit (Cat \# 52304; Qiagen, Hilden, Germany).

\section{RNA quantification and purity testing}

ThetotalRNAandits purityweremeasuredphotometrically using the NanoDrop [ND-1000 Spectrophotometer V3.1.0 (NanoDrop Technologies, Inc. Wilmington, DE USA. The optical density (OD) was measured at different wave lengths: $230 \mathrm{~nm}$ (absorption of contaminants \& background absorption), $260 \mathrm{~nm}$ (absorption maxima of nucleic acids), $280 \mathrm{~nm}$ (absorption maxima of proteins), and $320 \mathrm{~nm}$ (absorption of contaminants \& background absorption). The OD260/280 ratio was used as indicator for RNA purity. A ratio $>1.8$ was considered suitable for gene expression measurements ${ }^{[10,1]}$.

Reverse transcriptase polymerase chain reaction (RTqPCR)

This was done using high-Capacity cDNA Reverse Transcription Kit (applied biosystems ${ }^{\circledR}$ by life technologies ${ }^{\mathrm{TM}}$ ) and Veriti ${ }^{\circledR}$ 96-Well Thermal Cycler (applied biosystem ${ }^{\circledR}$ by life technologies ${ }^{\mathrm{TM}}$ ). Real time PCR amplification was done using (StepOnePlus ${ }^{\mathrm{TM}}$ ) and RT-qPCR System (applied biosystem ${ }^{\circledR}$ by life technologies ${ }^{\mathrm{TM}}$ ). Through the use of sequence specific primers for each target gene, the DNA polymerase from QuantiTeq SYBR Green PCR Master Mix amplified the target cDNA synthesized from RNA sample ${ }^{[12]}$. The primer sequences were as follows:

\section{HMGA2,}

Forward: 5'-TGGTGCAAGACTC-AGGAG-3'

Reverse: 5'-GCAGTCGGAAAGCAAAGG-3'[12]

MMP12,

Forward: 5'-GCAAGATTAACACAGGCATGG-3'

Reverse: 5'-AGCAGTGAGGAACA-AGTTGG-3'[12]

This step was divided into:

1. Preparation of PCR reaction mix: which was composed of $12.5 \mu \mathrm{L}$ of $2 \mathrm{X}$ Quanti Teq SYBR Green Master Mix $1 \mu \mathrm{L}$ of cDNA, $2 \mu \mathrm{L}$ of specific primer and 9.5 $\mu \mathrm{L}$ of nuclease free water. Glyceraldehyde-3-phosphate dehydrogenase (GAPDH) was the housekeeping gene, expression of which was used as internal standard. Non template control (NTC) sample containing water without cDNA was prepared with each run as negative control.

2. Loading the plate: the PCR reaction mix was transferred into each well of the reaction plate which was sealed and loaded into the instrument.

3. Running the RT-qPCR reaction: The plate document/ experiment for the run was created using the parameter values shown in (Table 1). Then the run itself was started $^{[13]}$. 
Table 1: PCR program cycles

\begin{tabular}{lcc}
\hline Step & Time & Temperature \\
\hline PCR initial heat activation & $2 \mathrm{~min}$. & $95^{\circ} \mathrm{C}$ \\
Denaturation & $15 \mathrm{~s}$ & $95^{\circ} \mathrm{C}$ \\
Annealing & $15 \mathrm{~s}$ & $60^{\circ} \mathrm{C}$ \\
Extension & $20 \mathrm{~s}$ & $72^{\circ} \mathrm{C}$ \\
Number of cycles & 45 & \\
\hline
\end{tabular}

From the amplification plot (Fig. 1 is an example), we set the baseline and threshold value to determine the threshold cycles (CT) for the amplification curves and we used a comparative method to analyze the data: the level of target gene expression (MMP-12 and HMGA2) was compared to that of the housekeeping gene (GAPDH) in biopsies I and II for each patient. Fold change of gene expression was determined according to the following equation:

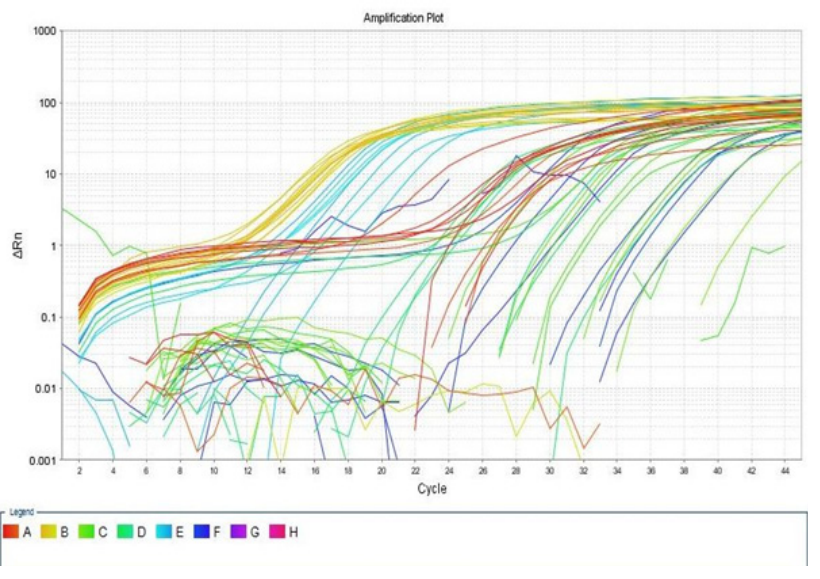

Fig. 1: Example of Amplification Plot (cases 1,2,16-23)

$\Delta \mathrm{CT}=\mathrm{CT}$ target $-\mathrm{CT}$ reference (house- keeping gene)

$\Delta \Delta \mathrm{CT}=\Delta \mathrm{CT}$ test sample $-\Delta \mathrm{CT}$ calibrator (control) sample

\section{$\mathrm{RQ}=\mathrm{Gene}$ expression $=\Delta \Delta \mathrm{CT}^{2[13]}$}

Statistical analysis: Data were coded and analyzed using Prism 7 for windows version 7.04 (C1992-2017 GraphPad Software, Inc). Descriptive statistics and Mann Whitney test were used to compare levels of gene expression between normal and tumor tissue groups. Chi square test was used to correlate level of expression of each gene with tumor degree of differentiation. The results were considered significant if $P$-value is $<.05$

\section{RESULTS}

One patient was excluded because target genes were detected neither in the tumor tissue nor in the normal tissue. Analysis of the results thus included only 23 patients; all of them were males in the age range between 49 and 78 years. All of them were smokers except one patient who quitted 7 years ago. All biopsies I were histologically positive for squamous cell carcinoma: 11 biopsies were highly differentiated, 10 biopsies were grade 2, and 2 biopsies were poorly differentiated. All biopsy II were reported as free from any tumor infiltration.

HMGA2 Gene : The minimum expression level of HMGA2 in normal tissue was 0.077 and in tumor tissue it was 0.63 while its maximum expression in normal tissue was 98.3 and in tumor tissue was 458 (Table 2). The median expression level of HMGA2 in the whole group was 0.98 in the normal tissue and 6.49 in the tumor tissue with a difference of 5.5. With the exception of two cases ( cases 5 and 18), there was higher relative expression of the HMGA2 gene in the tumor tissues compared to adjacent normal tissues (Fig. 2) and this was highly statistically significant $(P<0.0001)$. The more the level of expression of this gene, the poorer the tumor differentiation $(P<.0001)$

Table 2: Level of expression of HMGA2 in study group

\begin{tabular}{lcccc}
\hline Level of expression & Median & Minimum & Maximum & $P$-value \\
\hline $\begin{array}{l}\text { Normal tissue } \\
\text { sample }(\mathrm{n}=23)\end{array}$ & 0.98 & 0.077 & 98.3 & $<0.0001$ \\
Tumor tissue $(\mathrm{n}=23)$ & 6.49 & 0.63 & 458 & \\
\hline
\end{tabular}

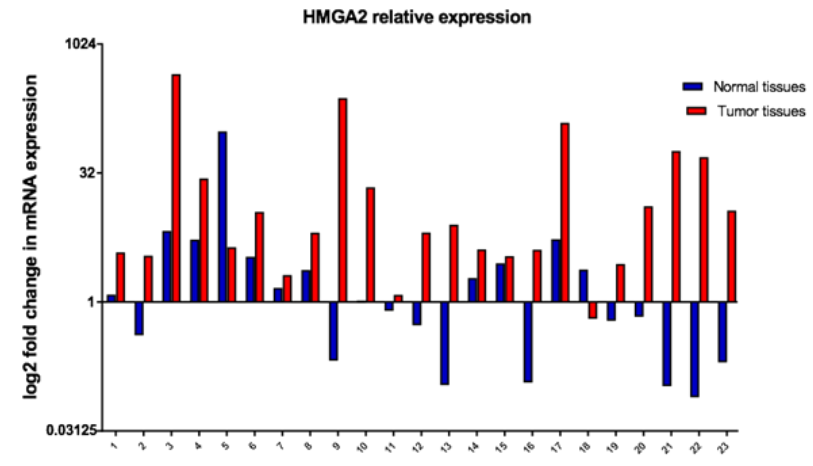

Patients'numbers

Fig. 2: Relative expression of HMGA2 in tumor tissue (red) and normal tissue (blue)

MMP12Gene: The minimum expression in normal tissue was 0.03 and in tumor tissue was 0.5 while the maximum expression of normal tissue was 83.1 and of tumor tissue was 9328 (Table 3). The median level of MMP12 was found to be 0.8 in the normal tissue and 122.6 in the tumor tissue with a difference of 121.7. With the exception of case no5 and case no 8 , there was higher expression of the MMP12 gene in the tumor tissues compared to adjacent normal tissues (Fig. 3) and this was highly statistically significant $(P$-value $<0.0001)$. The more the level of expression of this gene, the poorer the tumor differentiation $(P<.0008)$ 
Table 3: Level of expression of MMP12 Gene in study group

\begin{tabular}{lcccc}
\hline Level of expression & Median & Minimum & Maximum & P-value \\
\hline $\begin{array}{l}\text { Normal tissue } \\
\text { sample }(\mathrm{n}=23)\end{array}$ & 0.8 & 0.03 & 83.1 & $<0.0001$ \\
\begin{tabular}{l} 
Tumor tissue $(\mathrm{n}=23)$ \\
\hline
\end{tabular} & 122.6 & 0.5 & 9328 & \\
\hline
\end{tabular}

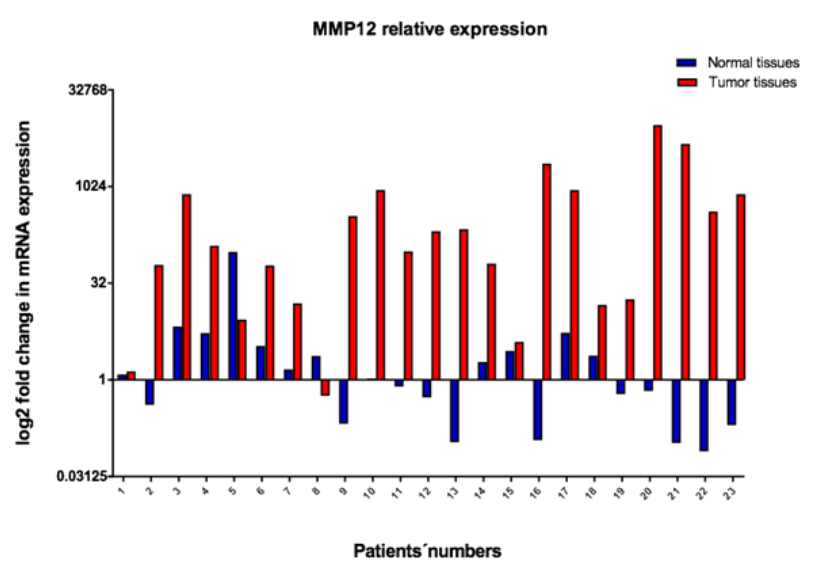

Fig. 3: Relative expression of MMP12 in tumor tissue (red) and normal tissue (blue)

\section{DISCUSSION}

With the exception of two cases, there was higher expression of the HMGA2 and MMP12 genes in tumor tissue compared to normal tissue and this was highly statistically significant $(P<0.0001)$. The more the level of expression of this two genes, the poorer the tumor differentiation.

Palumbo et al., studied the HMGA2 in 52 patients with esophageal cancer Ninety percent of their cases had overexpression of this gene in tumor tissue compared to normal tissues ${ }^{[14]}$. In LSCC two research groups similarly had overexpression of HMGA2 gene Järvinen et al., used a high resolution microarray technique to study expression of this gene in 20 patients with LSCC $^{[15]}$ and Li-Juan et al., used a complementary DNA microarrays in 8 patients with LSCC $^{[12]}$. Both studies had HMGA2 gene more expressed in tumor tissue than non-tumor tissue.

Fusco and Fedel reviewed the role on HMGA proteins in benign and malignant tumors and showed that its overexpression reflects cell transformation to neoplastic cells ${ }^{[16]}$. High expression of HMGA2 was also associated with tumor recurrence and poor prognosis. However, cancers with high expression of HMG2 had increased response to double strand breaks in DNA caused by radiation therapy and chemotherapeutic agents. This was considered a therapeutic advantage ${ }^{[17]}$.

Similar to our studies, other research groups found overexpression of MMP12 gene in head and neck tumors.
Impola et al., made a multicenter study in Japan, Finland and Canada on oral verrucous carcinoma $(\mathrm{n}=15)$ and SCC $(\mathrm{n}=15)^{[18]}$. In $90 \%$ of samples there was over-expression of MMP12. Kim et al, investigated the expression pattern of matrix metalloproteinases (MMPs) in 40 patients with HNSCC who had cervical node metastasis. MMP12 expression was found to be significantly associated with extracapsular spread (ECS) and correlated with nodal metastasis $(\mathrm{p}=0.024,0.011)$. No relation was found between MMP-12 expression and patient survival. Based on this , MMP-12 expressed in the primary tumor potentially could be used as a molecular marker to predict the presence ECS in HNSCC with nodal metastases ${ }^{[19]}$.

RT-qPCR is a highly sensitive test and any contamination of the normal tissue with clusters of cancer cells may obscure the difference in gene expression between tumor tissue and normal tissue. This may explain why tumor tissue in biopsies no. 5, 8 and 18 had less expression of the studied genes compared to normal tissue. Markowski et al., who studied gene expression profile in larygngeal cancer by high-density oligonucleotide microarrays; support this assumption as they also found 2 out 14 tissue samples contaminated with tumor tissue ${ }^{[20]}$.

The limitation of our study is the small sample size. Although all biopsies in our study, taken from normal tissue $1 \mathrm{~cm}$ away from tumor were free of tumor infiltration, it would have been better if we took a third biopsy further away from visible tumor. In this case we would have been able to study the spectrum of genetic alteration in the 'field of cancer' at large, particularly in less differentiated tumors. This may be the subject of our future research

Our current study may be the first in our country. Opening the area of gene expression in the LSCC helps to understand the molecular and cellular changes that take place in the tumor. This ultimately will reflect on the patient counseling and treatment.

In conclusion, expression of HMGA2 and MMP12 genes is higher in LSCC compared to normal tissue samples. The higher the level of expression of the two genes, the poorer the degree of differentiation of the tumor Further studies should be pursued to determine the diagnostic, therapeutic and prognostic implications of this in management of LSCC.

\section{CONFLICT OF INTERESTS:}

No conflict of interest to be declared.

\section{REFERENCES}

1. Stewart BW and Kleihues P. World Cancer Report. International Agency for Research on Cancer, WHO (2003). 232-6 
2. Lee KW, Kuo WR and Tsai SM. Different Impact from Betel Quid, Alcohol and Cigarette: Risk Factors for Pharyngeal and Laryngeal Cancer. Int J Cancer (2005). 117 (5):831-6

3. Jemal A, Murray T, Ward E, Samuels A, Tiwari RC, Ghafoor A, et al. Cancer Statistics. CA Cancer J Clin (2005). 55 (1):10-30

4. Almadori G, Bussu F, Cadoni G, Galli J, Rigante M and Artuso A. Multistep Laryngeal Carcinogenesis Helps Our Understanding of the Field Cancerisation Phenomenon: a review. Eur J Cancer (2004). 40 (16):2383-8

5. Choi P and Chen C. Genetic Expression Profiles and Biologic Pathway Alterations in Head and Neck Squamous Cell Carcinoma. Cancer (2005). 104 (6):1113-28

6. Pruitt KD, Tatusova T, Klimke W and Maglott DR. NCBI Reference Sequences: Current Status, Policy and New Initiatives. Nucleic Acids Res. 2009; 37(Database issue): D32-D36

7. Yamazaki H, Mori T, Yazawa M, Maeshima AM, Matsumoto F and Yoshimoto S. Stem Cell SelfRenewal Factors Bmi1 and HMGA2 in Head and Neck Squamous Cell Carcinoma: Clues for Diagnosis. Lab Invest. (2013). 93(12): 1331-8

8. Tatusova T. Ciufo S. Federhen S, Dedorov B, McVeigh R, O'Neill K et al. Update on Ref Seq Microbial Genomes Resources. Nucleic Acids Res (2015). 43(Database issue):D599-D605

9. Suzuki S, Sato M, Senoo $H$ and Ishikawa K. Direct Cell-Cell Interaction Enhances Pro-MMP-2 Production and Activation in Co-Culture of Laryngeal Cancer Cells and Fibroblasts: Involvement of EMMPRIN and MT1-MMP. Exp Cell Res (2004). 293 (2): 259-66

10. Manchester KL. Use of UV methods for measurement of protein and nucleic acid concentrations. Biotechniques (1996). 20 (6): 968-70

11. Gallagher SR and Desjardins PR. Quantitation of DNA and RNA with absorption and fluorescence spectroscopy. Curr Protoc Mol Biol (2006). 76 (1): Appendix. 3D 1- 3D 21

12. Li-Juan M,Wei L, Xin Z, Dong-Hai H, Hua Z, JianYun $X$, et al. Differential gene expression profiling of laryngeal squamous cell carcinoma by Laser capture microdissection and complementary DNA microarrays. Arch of Med Res (2009). 40 (2): 114-23
13. Livak KJ and Schmittgen TD. Analysis of relative gene expression data using real-time quantitative PCR and the 2(-Delta Delta CT) method. Methods (2001). 25(4):402-8

14. Palumbo Jr A, Da Costa NM, Esposito F, De Martino M, D'Angelo D, Leite de Sousa VP, et al. HMGA2 overexpression plays a critical role in the progression of esophageal squamous carcinoma. Oncotarget (2016). 7 (18): 25872-84

15. Järvinen AK, Autio R, Haapa-Paananen S, Wolf M, Saarela M, Grenman R, et al. Identification of target genes in laryngeal squamous cell carcinoma by high-resolution copy number and gene expression microarray analyses. Oncogene (2006). 25 (52): 6997-7008

16. Fusco A and Fedele M. Roles of HMGA proteins in cancer. Nat Rev Cancer (2007). 7 (12): 899-910

17. Boo LM, Lin HH, Chung V, Zhou B, Louie SG, O'Reilly MA et al. "High mobility group A2 potentiates genotoxic stress in part through the modulation of basal and DNA damage-dependent phosphatidylinositol 3-kinase-related protein kinase activation". Cancer Res (2005) 65 (15): 6622-30

18. Impola U, Uitto VJ, Hietanen J, Hakkinen L, Zhang L, Larjava H, et al. Differential expression of matrilysin-1 (MMP-7), $92 \mathrm{kD}$ gelatinase (MMP-9), and metalloelastase (MMP-12) in oral verrucous and squamous cell cancer. J Pathol (2004). 202 (1): 14-22

19. Kim JM, Kim HJ, Koo BS, Rha KS, Yoon YH Expression of matrix metalloproteinase-12 is correlated with extracapsular spread of tumor from nodes with metastasis in head and neck squamous cell carcinoma. Eur Arch Otorhinolaryngol. 2013 Mar;270(3):1137-42

20. Markowski J, Oczko-Wojciechowska M, Gierek T, Jarzab M, Paluch J, Kowalska M, et al. Gene expression profile analysis in laryngeal cancer by high-density oligonucleotide microarrays. $\mathrm{J}$ of Physiol and Pharmacol (2009). 60 (1): 57-63 